\author{
Pietro Folino-Gallo \\ Fabio Palazzo \\ Giuseppe Stirparo \\ Sergio De Filippis \\ Paolo Martelletti
}

\section{Price differentials of oral triptans in eight European Union countries}

P. Folino-Gallo • F. Palazzo • G. Stirparo Institute for Research on Population and Social Policies,

National Research Council, Rome, Italy

S. De Filippis • P. Martelletti ( $($ )

Headache Centre,

Department of Internal Medicine,

Sant'Andrea Hospital,

Second School of Medicine,

La Sapienza University,

Via di Grottarossa 1035-1039,

I-00189 Rome, Italy

e-mail: paolo.martelletti@uniroma1.it.

Tel.: +39-06-80345684

Fax: +39-06-80345323

\begin{abstract}
Triptans are presently a
milestone in the treatment of migraine patients. Because of their effectiveness and safety, they have radically improved migraine treatment but their use has meant a substantial increase in spending for medicines. We thus compared retail prices of triptans in eight European Union member states to establish the existence and the amount of price differentials. We found wide price differentials between countries (from $83 \%$ to $140 \%$ ) and within countries, where they attained $191 \%$ in Belgium. The least and most expensive products differed from country to country. These differentials mean
\end{abstract}

that the most cost-effective triptans differ from country to country and this can be an important source of variation in the treatment of migraineurs. A better-harmonised European system of pricing could limit these unethical variations.

Key Words Migraine - Triptans • Prices • European Union

\section{Introduction}

Since their introduction in early 1990s, selective 5HT1B/1D agonists (triptans) have radically improved migraine management because of their effectiveness, tolerability and safety. Their use, despite the high cost, has largely increased in the last years. Five different compounds (sumatriptan, naratriptan, zolmitriptan, rizatriptan and almotriptan) are listed in the 2002 WHO-ATC list [1] and thus are clinically available in at least one market. Eletriptan and frovatriptan are two newer triptans, and a total of seven different compounds will be soon available for treatment of migraine patients.

Because of the large number of clinically available triptans, physicians need comparative clinical information to select those products with the highest likelihood of clinical success as well as comparative cost information to prefer, when possible, those products with the best cost-efficacy ratio. Even if several studies have demonstrated the costeffectiveness of triptans, their use leads to substantial costs and there is a need, in a capped budget era, to limit expenditures without affecting quality of care.

Despite several reports comparing clinical efficacy of triptans and a meta-analysis comparing 53 clinical trials [2], no comparative data at the European level about triptan prices have been published and there is a gap of information at this level. Moreover, large price discrepancies between the European countries have been described for a variety of medicines. For all these reasons we thought it was of interest to compare costs of triptans and to study their price differentials. 


\section{Materials and methods}

Data were obtained from the EURO-Medicines database, a European Union-funded project aimed to collect information about medicines available in European countries and whose data are now available on the Internet (www.euromedicines.org). Details of the methodology and data sources used for collecting and analysing these data were provided elsewhere [3].

Our comparison refers to prices in 2000 and is limited to solid oral triptan formulations, which are licensed in all the EU countries and are more commonly used. Thus, suppositories and parenteral forms (injection and spray) were excluded from the comparison. Prices were compared using the cost per single unit (tablet, capsule, wafer), calculated by dividing the retail (pharmacy) price of the pack by the number of single units. Retail price includes ex-factory (industry) price, wholesale margin, pharmacist margin and value added tax (VAT); the extent of these different components differs from country to country.

Retail prices were calculated in local currency and converted in euro using the fixed conversion rate for 5 countries within the European monetary area (Belgium, France, Germany, Italy and Netherlands), or the September 2000 exchange rate for the 3 additional EU countries (1 euro equals 7.43 Danish crowns, 8.48 Swedish crowns and 0.63 British pounds). Incremental costs within countries were calculated assuming the cheapest price in each country as a reference.

\section{Results}

The lowest and highest prices, the countries with the lowest and highest prices and the percentage differences between countries are reported in Table 1 . The percentage differences between countries range, for a same compound, from $83 \%$ for rizatriptan $(5 \mathrm{mg}$ and $10 \mathrm{mg}$ ) to $140 \%$ for zolmitriptan (2.5 mg).

The triptans with the lowest price per unit by country are: naratriptan $2.5 \mathrm{mg}$ in Belgium (€ 5.00) and UK (€ 6.35); zolmitriptan $2.5 \mathrm{mg}$ in France (€ 4.69), Sweden (€ 5.88), UK (€ 6.35), Italy (€ 6.89) and Denmark (€ 7.52); and sumatriptan $50 \mathrm{mg}$ in The Netherlands (€ 4.62), Sweden (€ 5.59, parallel import), and Germany (€ 7.95).

The incremental costs per single unit of the triptans and of their different strengths are reported in Table 2. Naratrip$\tan 2.5 \mathrm{mg}$ is the least expensive triptan in Belgium and in the UK (together with zolmitriptan $2.5 \mathrm{mg}$ in the UK). There are some important differences between these two countries: (1) the price per tablet of naratriptan in the UK $(€ 6.35)$ is independent of pack size, while there is a $73 \%$ difference in Belgium according to the number of tablets in the pack; (2) the price of a zolmitriptan 2.5-mg unit is the same as that of a naratriptan 2.5-mg unit in the UK, while it is higher by

Table 1 Prices of solid oral triptan formulations in European countries

\begin{tabular}{lrllr}
\hline & Price range, euro & Country with the lowest price & Country with the highest price & Difference, \% \\
\hline Sumatriptan $50 \mathrm{mg}$ & $4.62-9.59$ & Netherlands & Denmark & 107 \\
Sumatriptan $100 \mathrm{mg}$ & $9.07-16.80$ & Netherlands & Denmark & 85 \\
Naratriptan $2.5 \mathrm{mg}$ & $5.00-11.32$ & Belgium & Germany & 127 \\
Zolmitriptan $2.5 \mathrm{mg}$ & $4.69-11.24$ & France & Germany & 140 \\
Rizatriptan $5 \mathrm{mg}$ & $6.21-11.36$ & Netherlands & Germany & 83 \\
Rizatriptan $10 \mathrm{mg}$ & $6.21-11.36$ & Netherlands & Germany & 83 \\
\hline
\end{tabular}

a Price per single unit (tablet, capsule or wafer)

Table 2 Incremental costs of triptan formulations within EU countries. Prices in each country were normalized to that of the least expensive formulation, set at " 100 ". Values are normalized prices or price ranges when there were differences due to package size

\begin{tabular}{|c|c|c|c|c|c|c|c|c|c|}
\hline & Belgium & Denmark & France & Germany & Italy & Netherlands & UK & Sweden & $\begin{array}{c}\text { Sweden } \\
\text { (parallel import) }\end{array}$ \\
\hline Sumatriptan $50 \mathrm{mg}$ & NA & $110-128$ & $108-118$ & $100-102$ & 101 & $100-105$ & $118-124$ & $106-132$ & $96-103$ \\
\hline Sumatriptan $100 \mathrm{mg}$ & $223-291$ & $214-223$ & NA & $179-204$ & 195 & 196-209 & 200 & $196-231$ & $176-190$ \\
\hline Naratriptan $2.5 \mathrm{mg}$ & $100-173$ & $102-108$ & $114-124$ & $116-142$ & NA & $141-145$ & 100 & $111-136$ & NA \\
\hline Zolmitriptan $2.5 \mathrm{mg}$ & $112-186$ & $100-126$ & $100-124$ & $116-141$ & $100-101$ & $117-120$ & 100 & $100-119$ & NA \\
\hline Zolmitriptan 5 mg & NA & NA & NA & NA & NA & NA & NA & $114-121$ & NA \\
\hline Rizatriptan 5 mg & 197 & $129-139$ & NA & $133-143$ & 101 & 134 & 111 & $106-139$ & NA \\
\hline Rizatriptan $10 \mathrm{mg}$ & $186-197$ & $120-139$ & NA & $133-143$ & 137 & 134 & 111 & $114-139$ & NA \\
\hline
\end{tabular}

$N A$, not available 
$12 \%-86 \%$ in Belgium; (3) the price of rizatriptan $5 \mathrm{mg}$, compared with naratriptan $2.5 \mathrm{mg}$, is $11 \%$ higher in the UK and $97 \%$ higher in Belgium.

Zolmitriptan $2.5 \mathrm{mg}$ is the least expensive triptan in Denmark, France, Italy, UK and Sweden (Table 2). There are no major differences in the cost of a single zolmitriptan 2.5-mg unit between different pack sizes in the UK and Italy, while these prices vary by $19 \%, 24 \%$ and $26 \%$ in Sweden, France and Denmark, respectively. The cost of a sumatriptan 50-mg tablet, as compared with zolmitriptan 2.5 $\mathrm{mg}$, is similar in Italy (1\%) but differs by $18 \%-24 \%$ in the UK. The cost of a rizatriptan 5- or 10-mg unit is higher than that of zolmitriptan $2.5 \mathrm{mg}$ by $11 \%$ in the UK, $1 \%-37 \%$ in Italy and 29\%-39\% in Denmark.

\section{Discussion}

From our data it appears that wide differentials in prices of triptans exist at a European Union level: the differences between countries for one compound range from $83 \%$ to $140 \%$ and they are not all in the same direction: the least expensive product in one country can be the most expensive in another country.

Moreover the extent of variations, excluding sumatriptan $100 \mathrm{mg}$, varies within countries from $24 \%$ in UK and France to $97 \%$ in Belgium. Including sumatriptan $100 \mathrm{mg}$ the extent of variation becomes much wider, attaining $100 \%$ and $191 \%$ in the UK and Belgium, respectively.

Selection of the right triptan for an individual patient is a complex choice: it must take in consideration at least the clinical characteristics of the single patient (frequency and severity of migraine attacks, risk factors, contraindications to treatment), the efficacy of each triptan (pain-free response at $2 \mathrm{~h}$, sustained pain-free rates, and recurrence rates), and the costs of treatment.

The wide price differentials mean that the most costeffective triptan can differ in Europe from country to country and this is a source of variation in the treatment of migraineurs. A better-harmonised European system of pricing could limit these unethical variations.

\section{References}

\author{
1. - (2002) WHO Collaborating Centre \\ for Drug Statistics Methodology. ATC \\ code 2002, WHO-CC, Oslo
}

2. Ferrari MD, Roon KI, Lipton RB, Goadsby PJ (2001) Oral triptans (serotonin 5-HT(1B/1D) agonists) in acute migraine treatment: a meta-analysis of 53 trials. Lancet 358:1668-1675
3 Folino-Gallo P, Walley T, Frolich JC, Carvajal A, Edwards IR (2001) Availability of medicines in the European Union: results from the EURO-Medicines project. Eur J Clin Pharmacol 57:441-446 